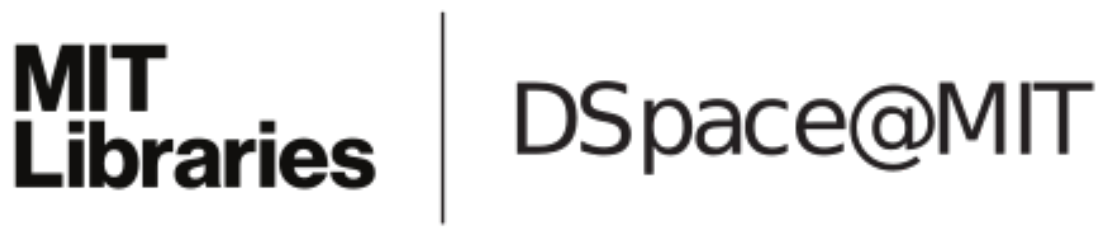

\author{
MIT Open Access Articles
}

Multi-electrode Harmonized Kingdon Traps

The MIT Faculty has made this article openly available. Please share how this access benefits you. Your story matters.

As Published: https://doi.org/10.1007/s13361-018-2032-9

Publisher: Springer US

Persistent URL: https://hdl.handle.net/1721.1/131923

Version: Author's final manuscript: final author's manuscript post peer review, without publisher's formatting or copy editing

Terms of Use: Article is made available in accordance with the publisher's policy and may be subject to US copyright law. Please refer to the publisher's site for terms of use. 


\title{
MULTI-ELECTRODE HARMONIZED KINGDON TRAPS
}

Evgeny Nikolaev ${ }^{1}$, Mikhail Sudakov ${ }^{1,3}$, Gleb Vladimirov ${ }^{1}$, Luis Fernando Velásquez-García ${ }^{2}$, Petr Borisovets $^{1}$, Anastasia Fursova ${ }^{1}$

1 Skolkovo Institute of Science and Technology, Moscow, Russian Federation

2 Microsystems Technology Laboratories, Massachusetts Institute of Technology, Cambridge MA, USA

3 Ryazan Radiotechnical University, Ryazan, Russian Federation

*Correspondence to e.nikolaev@skoltech.ru

\begin{abstract}
Based on the analysis of the results of the study of various designs of multi-electrode harmonized Kingdon traps, we propose a new type of trap with two merged internal electrodes that has the ability to capture and accumulate ions formed inside. We also investigated the influence of inaccuracies in the manufacture of the electrodes on the field inside such trap. The four-electrode trap, which actually degenerates into a two-electrode device with traces of two other electrodes present at the ends of the internal electrodes (their splitting) has been found as the less sensitive to inaccuracies caused by manufacturing and cutting the ends of trap electrodes. We show that a mass spectrometer with a relatively high resolving power can be created on the basis of such a trap. The creation of the traps requires the manufacture of complex electrodes with demanded accuracy of their surfaces. This becomes possible with the advent of 3D printers.
\end{abstract}

\section{Introduction}

The Kingdon ion trap, invented in the beginning of the 20th century, has proved to be of great relevance for mass spectrometry. The original Kingdon trap [1] is a cylinder (external electrode) with closed ends that have holes to hold a wire (internal electrode) stretched along the entire cylinder along its axis. The cylinder is grounded, and a negative electric potential is applied to the wire for confining positive ions (a positive bias voltage would be used for confining negative ions). The ions created with some non-zero momentum inside such a system do not fall on the wire because of their momentum conservation, and do not strike the surface of the cylinder because their total energy (the absolute value of which is between the magnitude of the wire potential and the potential of the cylinder) is less than the surface potential of the cylinder. The most famous Kingdon trap is a device used in mass spectrometers invented by A. Makarov and manufactured by Thermo under the name Orbitrap, which has been extensively investigated and described [2]. However, very little is known about other types of Kingdon traps, although the possibilities for their creation have been investigated by Professor Golikov and his collaborators more than a decade ago [3]. Among them, there are many-electrode Kingdon traps with a quadratic potential in one of the directions, which we call in this work the harmonized Kingdon traps. In this terminology, the Orbitrap trap is a one-electrode harmonized Kingdon trap.

The Orbitrap is a further development of Knight's ideas -the first to propose measuring the frequencies of ions oscillating in a quadratic field to measure their masses, and also suggested an electrostatic trap for creating such a field [4]. A feature of the quadratic field is that the frequency of the motion of the ions does not depend on the amplitude of their oscillations, and is inversely proportional to 
the square root of the ion mass-to-charge ratio. Mass spectrometers based on Kingdon traps could be of great interest as portable devices because the trap itself is purely electrostatic and low energy consuming mass analyzer. Nevertheless, if we consider Orbitrap's trap as a core for such mass spectrometer we will find two fundamental drawbacks that compelled its creators to propose a more complex ion manipulation system for creating a mass spectrometer, namely $(i)$ the need to trap accelerated ions with a shift from the center of the trap, and (ii) the need for a pulsed high voltage supply to control the electric potential of the central electrode. The Orbitrap cannot capture the ions created inside; therefore, the trap can only be used in conjunction with another trap (e.g., C-trap in the implemented Orbitrap-based mass spectrometer [2]) or a pulsed ion source. The use of an additional radio frequency trap with a complex system of deflectors to inject the ions to the Orbitrap, as well as the use of a high-voltage pulsed power supply, significantly increases the energy consumption of the device, and also greatly complicates its implementation as a portable device or as scientific payload for space applications.

However, it is possible to create harmonized Kingdon traps that hold the ions created inside them and allow measuring their masses. While analyzing the solutions of the Laplace equation with the goal of creating time-of-flight mass spectrometers with time focusing of infinitely high order, Golikov found a family of Kingdon traps with several internal electrodes [3],[5]. Golikov's work shows that there can be any number of internal electrodes stretched parallel to the axis of the external electrode without interfering with the creation of a quadratic potential in the space between them along the direction of their extension. Of course, in order to create such a quadratic potential, the geometry of the external electrode is more complex than a cylinder of fixed radius, which was found by Golikov for a range of traps with different number of internal electrodes. In this work, we use modeling and simulations to investigate a plurality of four-electrode harmonized Kingdon traps to determine their sensitivity to the manufacturing and assembly errors of electrodes, as well as to the fringing fields caused by the truncation of the trap. From this effort, we identified that the harmonized Kingdon traps with two merged internal electrodes have the lowest sensitivity to these distortions in geometry. We synthesized a design of such a trap, investigated its ability to capture and accumulate ions formed inside, and explored the use of such a trap as a mass spectrometer.

\section{Modeling and design of four-electrode harmonized Kingdon traps}

In the work of Golikov's group, e.g. [6], and also in the paper of Klaus Köster [7], variants of the Kingdon ion trap with multiple internal electrodes and harmonic potential on a given direction are described. These traps were first described in the papers and dissertations of the Golikov's group, which unfortunately are not available to a broad international audience since they are written in Russian. Köster, apparently independently, proposed a Kingdon trap with two internal electrodes, which he named Cassinian trap because its electric field can be described in part by the Cassinian equation. Köster modeled, implemented, and characterized experimentally his Cassinian trap, obtaining resolution values close to those demonstrated on Orbitrap prototypes; he also indicated ways to increase the resolving power and other characteristics of the instrument.

In the multi-electrode harmonic Kingdon traps, as in the Orbitrap, the distribution of the field is quadratic in one of the directions (usually along the axis of the electrodes, i.e., the $z$-axis). The motion of ions in this direction is a harmonic oscillation in which the oscillation frequency exclusively depends on the mass-to-charge ratio of the ions; therefore, through the measurement of the oscillation frequency, it is possible to determine the mass of ions, which makes possible to use such traps as mass spectrometers (in such multi-electrode harmonized Kingdon traps, the signal induced by the oscillating ions on the external 
electrode is measured, as is done in Orbitrap, to obtain the mass spectra via a Fourier transform). The electric potential distribution of a four-electrode harmonized Kingdon trap (Fig. 1), i.e., $\varphi(x, y, z)$, is obtained by solving the Laplace equation using as constraint the quadratic dependence of the potential on one of the coordinates; the result is the expression:

$$
\begin{aligned}
& \varphi(x, y, z)=c\left(z^{2}-\frac{c x^{2}+d y^{2}}{2}\right)+\ln \left[\left((x+a)^{2}+(y+b)^{2}\right)\left((x+a)^{2}+(y-b)^{2}\right)\right]+ \\
& \ln \left[\left((x-a)^{2}+(y+b)^{2}\right)\left((x-a)^{2}+(y-b)^{2}\right)\right]
\end{aligned}
$$

where $x$ and $y$ are directions perpendicular between them and to the axis of the trap, i.e., $z$, and $a, b, c$, and $d$ are constants. Here we use formalism and ideas of Golikov's group [6]. In this kind of trap, in addition to the orbital rotation of ions around the internal electrodes, orbital rotations around subsets of the internal electrodes are possible, as shown in Fig. 2. After calculating the potential and shape of the electrodes (which follow equipotential surfaces), the solution can be normalized so that the external electrode has a potential of zero, and the bias voltage of the internal electrode is equal to -1 . The choice of the equipotentials for positioning the internal and external electrodes was made on the bases of two demands: first, not to create electric field on the surface of these electrodes strong enough to cause autoemission of electrons and second, to create space to trap enough ions for high dynamic range. Therefore, the actual solution is the normalized solution multiplied by the required voltage of the internal rods, which in this example was $4 \mathrm{kV}$. In Fig. 2, the trajectories of the ions were estimated using the SIMAX program [8], which can receive as input electric potential solutions from the SIMION program [9] and produce accurate particle tracing in these fields. In Fig. 2, left, a $500 \mathrm{Da}$ ion starts from the point $x_{o}=20 \mathrm{~mm}, y_{o}=$ $20 \mathrm{~mm}, z_{o}=0 \mathrm{~mm}$ with energy $2,000 \mathrm{eV}$ along the $y$ axis; similarly, on Fig. 2, right, the same ion starts from the point $x_{o}=13.5 \mathrm{~mm}, y_{o}=13.5 \mathrm{~mm}, z_{o}=0 \mathrm{~mm}$, having an initial energy of $500 \mathrm{eV}$ along the $y$-axis and $500 \mathrm{eV}$ along the $x$-axis. Note that the oscillations along the $z$-axis are harmonic and strictly isochronous, with a frequency that depends only on the mass-to-charge ratio of the ions.

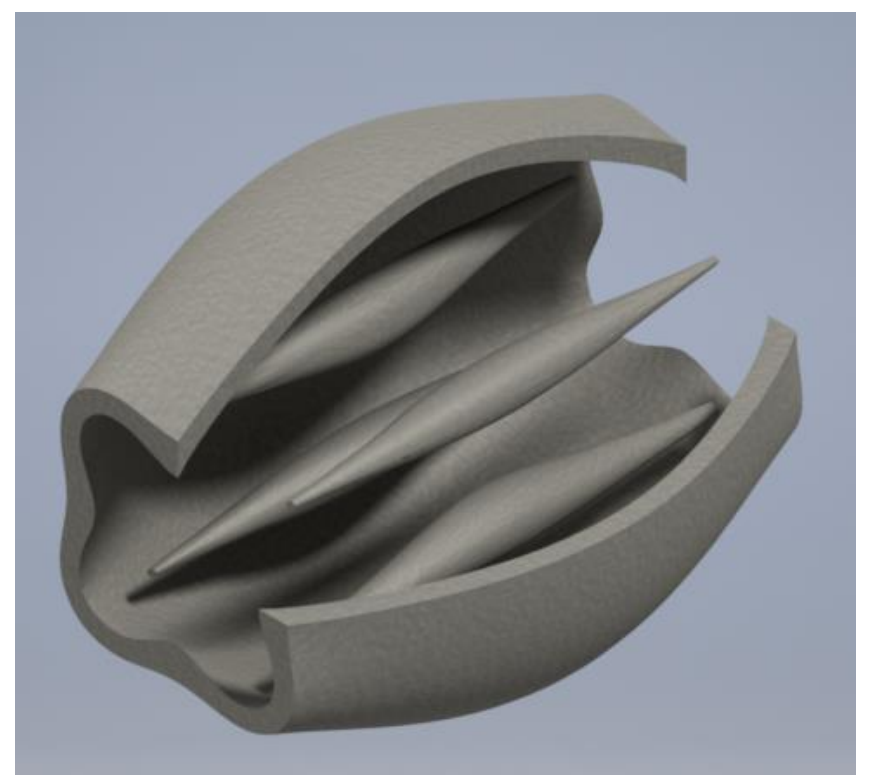

Fig.1. An example of a harmonized Kingdon trap with four internal electrodes. The schematic shows only the bottom pair of internal rods, above them there is another pair. In this example $a=0.9, b=0.9, c=$ 
$0.2, d=0.5$, the external electrode is biased at $U_{\text {high }}=7$, and the internal electrodes are biased at $U_{\text {low }}=$ 1 .

Four-electrode harmonized Kingdon traps can also be obtained as a superposition of two Cassinian traps. The internal electrodes of the two two-electrode traps can be rotated relative to each other at an arbitrary angle around the $z$-axis. At small angles between the planes in which the pairs of Cassinian traps lie, the electrodes will merge, resulting in a trap with two internal electrodes with more complex shapes than the electrodes of the original Cassinian traps (in the next section such traps will be described in more detail). We investigated a variety of four-electrode Kingdon traps, primarily to determine their sensitivity to the manufacturing and assembly errors of electrodes, as well as to the fringing fields caused by the truncation of the trap; such investigations are necessary because the non-ideality of the electric field (i.e., its difference from the quadratic term and $(x, y)$ dependence of the coefficient that multiplies the quadratic term in the polynomial equation that describes the dependence on $z$ of the electric potential in the volume in which the ion motion takes place) leads to a rapid dephasing of the harmonic oscillations along the $z$-axis, which leads to loss of resolving power. Investigations of the influence of electrode roughness can be done in the following way. In SIMION software a uniform grid is used to present field distributions and electrodes are presented by this grid with the same voltage across the electrode. With curved electrodes the grid points do not fall exactly on the boundaries of electrodes. This can be treated as electrode with not even, distorted shape. We produced such potential arrays with a grid step $0.075 \mathrm{~mm}$ for both Cassinian trap with two rods and for our geometry and refined them. Those arrays were used further in simulation with measurement of induced ion current. According to our simulations dephasing of the ion clouds due to mechanical roughness of electrodes of 75 microns happens within $10 \mathrm{~ms}$ for Cassinian ion trap with two rods and within more than $20 \mathrm{~ms}$ for our geometry with merged rods. The lion trap with merged electrodes have addition degree of freedom to change the shape of the rods in order to make geometry less sensitive to mechanical distortions of electrodes. Our trap could be optimized further in case of necessity.

Our simulation results show that the traps with two internal merged electrodes have the lowest sensitivity to these distortions in geometry. The parameters used in the optimal solution are $a=0.1, b=$ $0.73, c=2$, and $d=0$; the shape of the external electrode, (which is determined by the shape of the equipotential surface for the selected potential), was given by the condition $U_{\text {high }}=2.0$, and the bias voltage acting on the internal electrodes is $U_{\text {low }}=-2.8$. The trap has a dimension along the $z$-axis of 66 $\mathrm{mm}$-all the electrodes are extended $3 \mathrm{~mm}$ at both ends using cylinders with diameters that coincide with the electrode diameters at their cutoff point to correct the fringing fields near the ends. A 3D view of such a trap is given in Fig. 3. In this design, the openings at the ends of the traps, formed because of its cutting, are close to the minimum possible dimensions; therefore, the influence of the penetration of fields from external structures through these holes is minimal. 


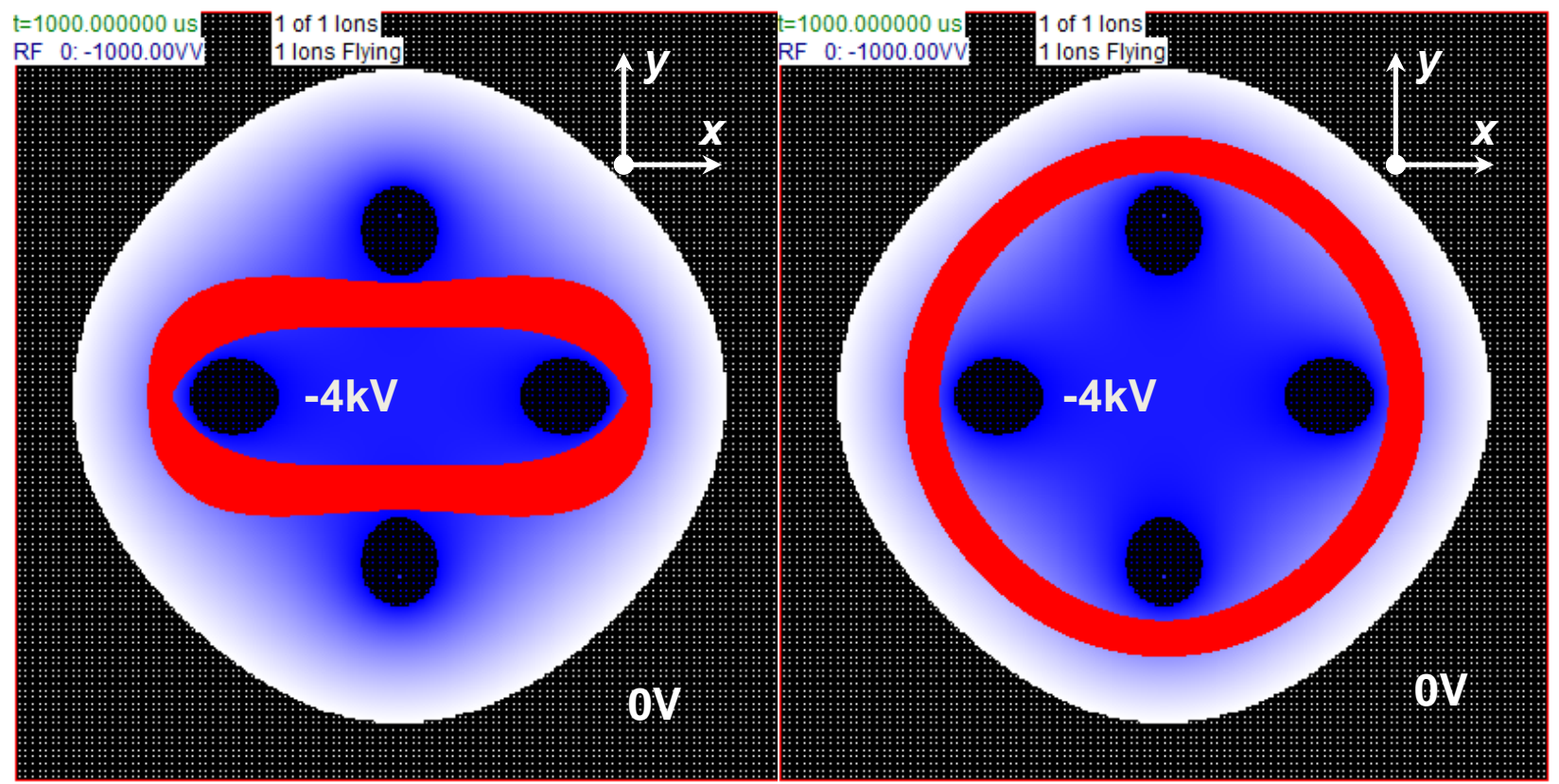

Fig.2. An example of ion orbital rotation around all the internal electrodes (left) and around two internal electrodes (right) in a four-electrode harmonized Kingdon trap; the ions also oscillate along the z-axis due to a quadratic potential in $z$. The figures show projection of ion trajectories on the transverse crosssection of the trap at $z=0$. When the ions are introduced from the outside, it is necessary to momentarily increase the bias voltage at the internal electrodes with a pulse to reduce their kinetic energy to the required value for orbital capture -otherwise, the ions will collide with the outer electrode due to energy conservation. The ions are not introduced into the trap at the center $(z=0)$, but at some distance from the center along the z-axis, which initiates the oscillations of the cloud along the z-axis. The location of ion injection along the z-axis defines the amplitude of the oscillations.

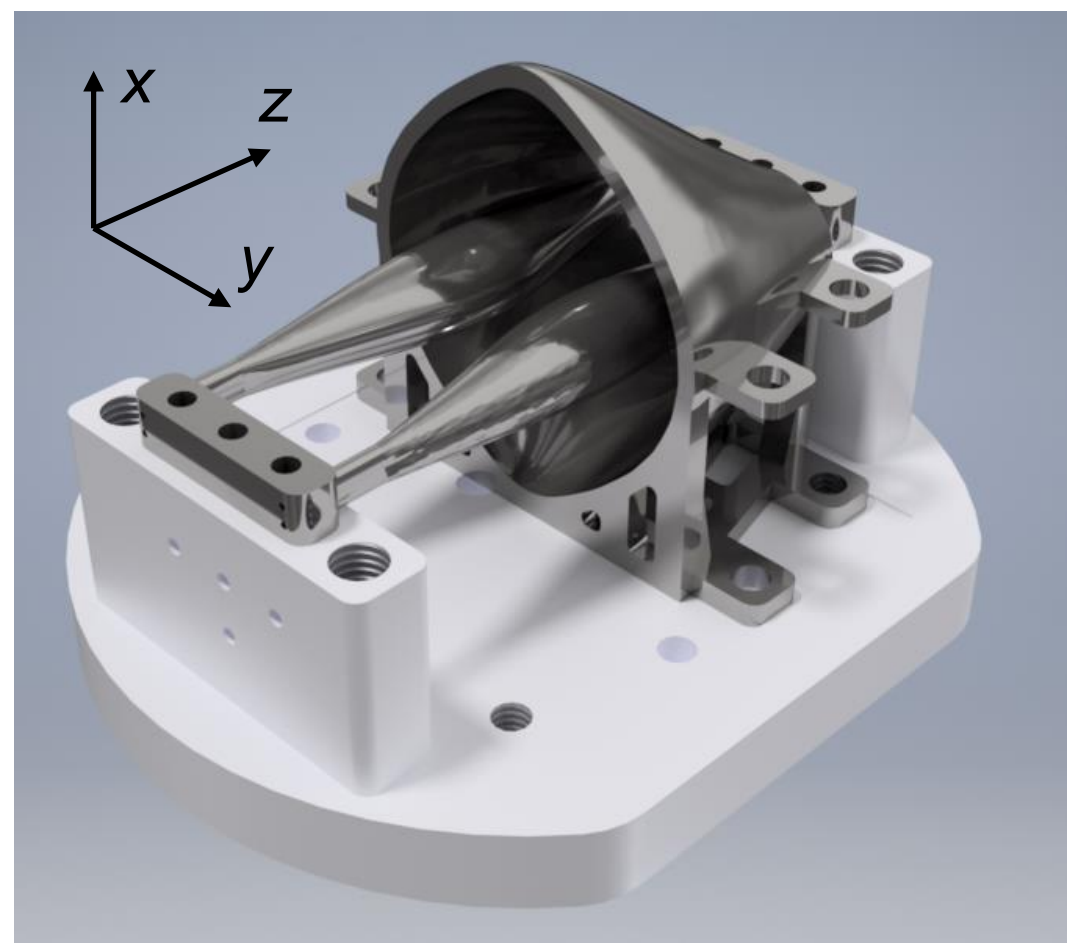


Fig.3. 3D schematic of a four-electrode harmonized Kingdon trap with two merged internal electrodes.

\section{Difference between Refined and Analytical fields}

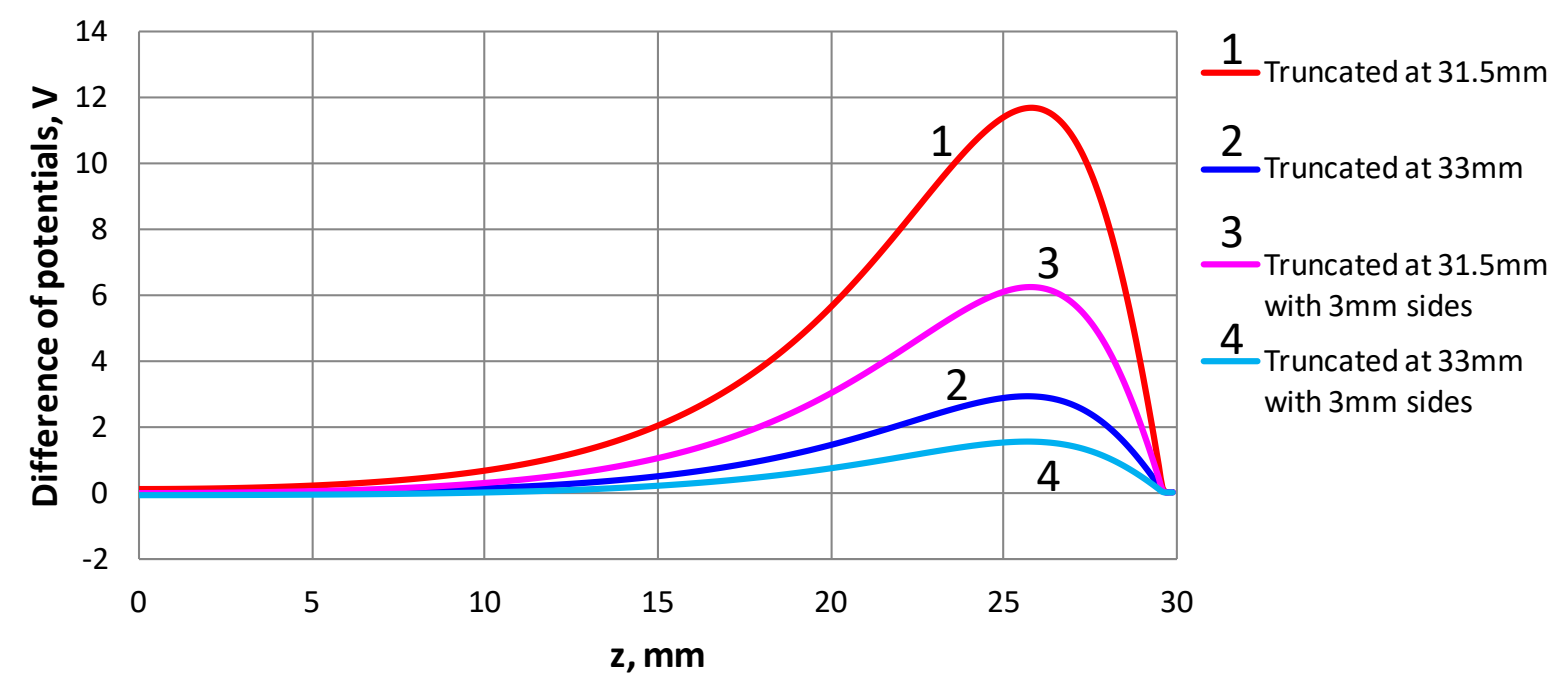

Fig. 4. Difference between the distribution of the actual electric potential along the $z$-axis (for $x=0, y=$ 0 ) and that of the ideal trap for different truncation variants (see the legend). The internal electrodes are biased at a potential of $4 \mathrm{kV}$ while the external electrode is grounded. Here "sides" are additional $3 \mathrm{~mm}$ $2 D$ sections that are added from both sides of the trap at the place of truncation. Shape of electrodes in those $2 D$ sections repeat the shape of truncated electrodes.

Figure 4 shows the difference in the distribution of the electric potential along the $z$-axis from the ideal (i.e., calculated analytically using equation (1)) for different values of the $z$ coordinate at which the trap is cut off. It is noticeable that, in all cases, the addition of extensions on both ends of the trap reduces the difference; the lowest of the graphs in Fig. 4 (corresponding to the cutoff at $33 \mathrm{~mm}$ from the center of the trap with $3 \mathrm{~mm}$ added extensions) indicates a nearly complete suppression of the fringing fields near the center of the trap. Although the graphs in Fig. 4 show the behavior of the deviation of the potential from the ideal along the $z$-axis, the curves are not entirely informative in terms of evaluating the nonlinear distortions. The deviations calculated in Fig. 4 contain a constant component that does not change the motion of ions at all, a quadratic component that only slightly changes the quadratic potential (and hence only the absolute value of the oscillation frequency), and higher-order terms that carry information about nonlinear deviations (the dependence of the oscillation frequency on their amplitude). To calculate the latter, we can use the method of polynomial regression, but before proceeding to this, we calculate the amplitude of the quadrupole component for the unperturbed field. To this end, we represent the potential along the $z$-axis, i.e., $\varphi(z)$ in the form

$$
\varphi(z)=U_{\text {rods }} A_{2}\left(\frac{z}{z_{0}}\right)^{2}
$$

where $U_{\text {rods }}$ is the bias voltage on the internal electrodes with a grounded external electrode, $z_{o}=33 \mathrm{~mm}$, i.e., the distance from the center of the trap to the cut ends, and $A_{2}$ is the amplitude of the quadrupole field. The equation of motion of ions with mass $m$ and charge $e$ in such a potential has the form 


$$
m \frac{d^{2}}{d t^{2}} z=-e \cdot U_{\text {rods } s} A_{2} \frac{2 z}{z_{o}^{2}},
$$

which describes harmonic oscillations with an angular frequency $\Omega$ equal to

$$
\mathbf{\Omega}=\sqrt{\frac{2 e \cdot U_{\text {rods }} A_{\mathbf{2}}}{m z_{o}^{2}}}
$$

The simulation of this system showed that for singly-charged ions of mass $500 \mathrm{Da}$, the frequency of axial oscillations is $f=186,690 \mathrm{~Hz}$. From this value, we can calculate the amplitude of the quadrupole field as

$$
A_{2}=\frac{m z_{o}^{2}}{2 e \cdot U_{\text {rods }}}(2 \pi f)^{2}=0.971 ;
$$

the value is close to unity and it will serve us as a basis for comparison with the amplitudes of nonlinear distortions, which are calculated below.

As already mentioned above, it is convenient to use a polynomial regression to calculate the amplitudes of nonlinear distortions. In this case, it is necessary to take into account two circumstances. First, the interpolation should be built in the region where the ions move while being trapped (in the next section, it will be shown that this is the region near the center of the trap $-6 \mathrm{~mm}<z<6 \mathrm{~mm}$ ). Secondly, we should not use higher-order polynomials for interpolation, since the amplitudes of higher polynomials increase rapidly. In this study we used polynomials up to the sixth order inclusive. Thus, the interpolation polynomial for the potential distribution along the $z$-axis has the form

$$
\varphi(z)=\sum_{n=0}^{6} U_{n}\left(\frac{z}{z_{0}}\right)^{n}
$$

At the line starting at the point $x=0, y=0$, the interpolation polynomial contains only even powers of $z$ because of the symmetry of the distribution with respect to the center of the trap. The calculations using the MathCad 13 program [10] yielded the amplitudes given in Table 1. The amplitudes are not significant, but the change in the amplitudes of the higher distortions shows that the best trap of the set is trap number 3. Here numbering of geometries of traps in the first column of the table correspond to figure 4, namely $1^{\text {st }}$ and $2^{\text {nd }}$ rows are traps truncated at the distance $31 \mathrm{~mm}$ and $33 \mathrm{~mm}$ from the trap centre and without $2 \mathrm{D}$ sections added from both sides of truncation. Rows 3 and 4 are the same traps as 1 and 2, but with additional 2D sections added from both sides of truncation. The fifth row corresponds to a trap truncated at $33 \mathrm{~mm}$ from trap centre and with coarse electrode surfaces of accuracy $0.075 \mathrm{~mm}$.

Table 1. Dimensionless amplitudes of the distortions of the axial electric potential for traps of the four types of four-electrode harmonized Kingdon traps described in Fig. 4 (the fifth row corresponds to a trap with coarse electrode surfaces, as described in the text). 


\begin{tabular}{|c|c|crrr|}
\hline Trap type & Zo, $\mathrm{mm}$ & \multicolumn{1}{|c}{ U0 } & U2 & \multicolumn{1}{c|}{ U4 } & \multicolumn{1}{c|}{ U6 } \\
\hline \multirow{2}{*}{1} & 31 & 0.097 & 4.185 & 14.661 & 95.395 \\
2 & 32 & -0.019 & 2.403 & 3.105 & 128.546 \\
\cline { 2 - 3 } 3 & 31 & -0.031 & 1.184 & -0.586 & 96.297 \\
4 & 32 & -0.032 & 0.733 & -3.671 & 100.597 \\
\cline { 2 - 3 } 4 & 32 & -8.552 & 20.869 & -403.341 & 7207 \\
\hline 5 & & &
\end{tabular}

We also investigated the influence of inaccuracies in the manufacture of the electrodes on the field inside the trap. These studies were performed using the SIMION program. The surface of each electrode is an equipotential; given that in SIMION the electrode is set on a uniform grid, the nodes of which do not fall on the curved surface of each electrode can be treated as an unevenness of the electrode surface. Fig. 5 shows the difference between the exact potential and the potential given by SIMION using its finest mesh size for mapping electrodes (a grid spacing of $75 \mu \mathrm{m}$ ); the amplitudes of the nonlinear distortions for this case are given in the fifth row of Table 1 -the result not only reflects the effect of the inaccuracy in the manufacture of electrodes, but also the edge effects due to electrode cutoff. The nonlinear amplitudes in this case are quite large compared to the other cases examined; however, considering that the electrodes can be manufactured with accuracy not worse than $5 \mu \mathrm{m}$ using precision machining, these values will be an order of magnitude smaller and should not significantly affect the parameters of the device. 


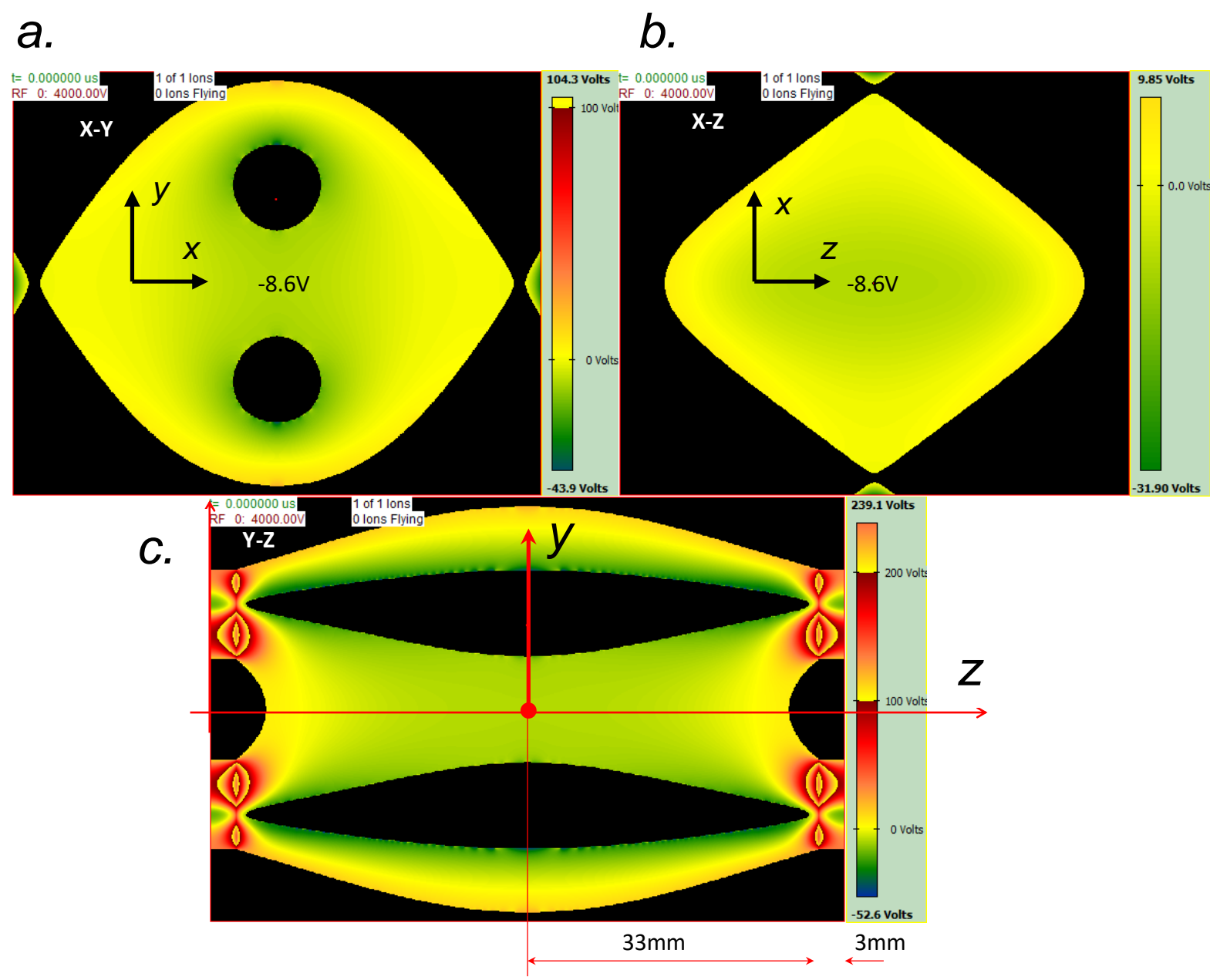

Fig.5. $x-y(a), x-z(d)$, and $y-z(c)$ sections (not in scale)) of the difference between the electric potentials of an ideal trap and a trap with electrodes with coarse surface (due to the discretization of the shapes using a $75 \mu \mathrm{m}$ pitch grid). The external electrode is grounded, the bias voltage on the internal electrodes is $-4 \mathrm{kV}$. Notice that the color bar resets for values larger than $100 \mathrm{~V}$ and $200 \mathrm{~V}$.

For the final design of the trap, shown in Fig. 5, we carried out a more detailed study of the deviation of the axial distribution of the potential from the ideal case due to the cutoff of the trap along the ends. In this case, the difference in the potential distribution along the $z$-axis was calculated for the ideal trap and truncated along the lines at constant $(x, y)$ values; these functions were then subjected to polynomial interpolation (i.e., equation (6)). The study showed that at distances from the axis of the trap in the $x$-direction over $20 \mathrm{~mm}$, the difference between the distributions of the ideal trap and the cutoff trap is negligible. This is understandable since the edge effects in this region are negligible as well. For other lines, the results are shown in Table 2.

\begin{tabular}{|c|c|c|c|c|c|c|c|c|}
\hline $\mathbf{x}(\mathbf{m m})$ & $\mathbf{y}(\mathbf{m m})$ & $\mathbf{U}_{\mathbf{0}}$ & $\mathbf{U}_{\mathbf{1}}$ & $\mathbf{U}_{\mathbf{2}}$ & $\mathbf{U}_{\mathbf{3}}$ & $\mathbf{U}_{\mathbf{4}}$ & $\mathbf{U}_{\mathbf{5}}$ & $\mathbf{U}_{\mathbf{6}}$ \\
\hline 0 & 0 & $-1.700 \mathrm{E}-2$ & $0.000 \mathrm{E}+0$ & $5.780 \mathrm{E}-1$ & $0.000 \mathrm{E}+0$ & $3.270 \mathrm{E}+0$ & $0.000 \mathrm{E}+0$ & $8.915 \mathrm{E}+1$ \\
15 & 0 & $-6.500 \mathrm{E}-2$ & $0.000 \mathrm{E}+0$ & $6.950 \mathrm{E}-1$ & $0.000 \mathrm{E}+0$ & $-6.998 \mathrm{E}+0$ & $0.000 \mathrm{E}+0$ & $1.018 \mathrm{E}+2$ \\
15 & 15 & $-6.300 \mathrm{E}-2$ & $1.800 \mathrm{E}-3$ & $7.820 \mathrm{E}-1$ & $-2.716 \mathrm{E}+0$ & $1.222 \mathrm{E}+1$ & $-2.685 \mathrm{E}+1$ & $1.967 \mathrm{E}+1$ \\
\hline
\end{tabular}


Table 2. The values of the amplitude of the deviations of the ideal trap field from the truncated along various straight lines $(x, y)$ in the regions where ions move.

The calculations given in Table 2 show a rather significant change in the value of $U_{2}$, which changes the frequency of the axial oscillations of the ions. However, this would happen if the motion of ions along the $z$-direction would occur at constant values of $x$ and $y$; in reality, as follows from zoscillation frequency spectra (see next section), when the ion moves along $z$, oscillations along the $x$ - and $y$-directions also occur. In this case, the values of $U_{2}$ and the values of the other nonlinear amplitudes $U_{n}$ leverage. As a result, the dependence of the ion oscillation frequency along the $z$-direction weakly depends on the initial position of the ion. This was proved by calculating the oscillation frequencies of ions with a mass of $50 \mathrm{Da}$ started from the position $z_{0}=6 \mathrm{~mm}$ and different positions $(x, y)$ having zero initial energy, which resulted in harmonic oscillations of the ions along the $z$-direction in the $-6 \mathrm{~mm}$ to 6 $\mathrm{mm}$ range. The frequencies were calculated by Fourier analysis of the signal produced by these oscillations. We did not find any differences in the frequency of the ion oscillation within an error of 1 Hz.

\section{Multi-electrode harmonized Kingdon traps with merged internal electrodes}

A practical multi-electrode harmonized Kingdon trap has electrodes of finite dimensions with surfaces that coincide, as much as possible, with equipotential surfaces of the ideal electric field solution; evidently, the shape and size of these electrodes are determined by the choice of the potential that is applied to them. When selecting their geometry, the electrodes must be positioned so that they do not interfere with the motion of the trapped ions, that is, they should not be in the regions of stable ion motion. In the case of multi-electrode harmonized Kingdon traps, when these conditions are met, the electrodes can merge and, as already mentioned, instead of four internal electrodes, two electrodes of a more complex geometric shape result (Fig. 3). The four-electrode trap actually degenerates into a twoelectrode device with traces of two other electrodes present at the ends of the internal electrodes (their splitting). The advantage of this design is that the cut ends of the outer electrode practically cover the inner region of the trap and the penetration of the field from the external electrodes, resulting in minimal distortions of the fields. At the same time, the internal electrodes are of sufficient thickness to be technically feasible to anchor them outside the trap. 
a.

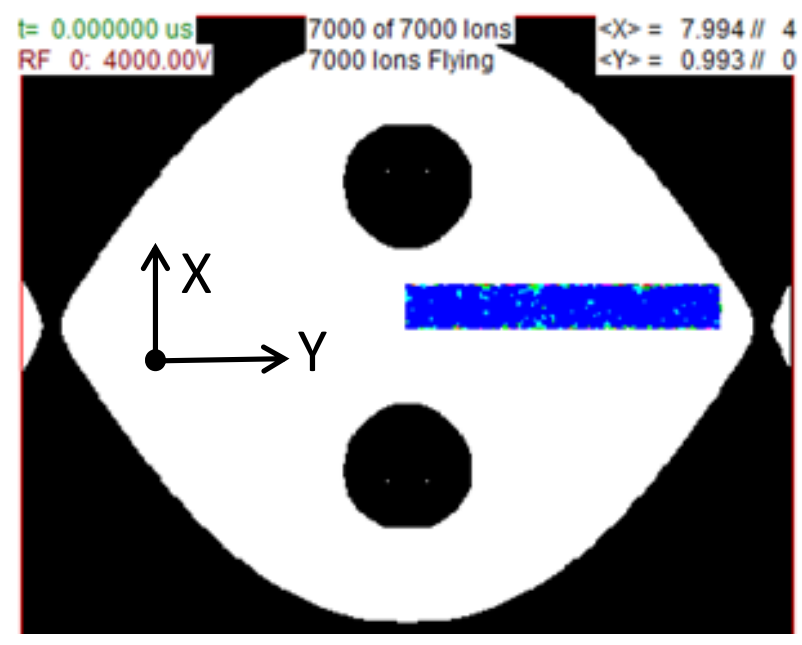

b.

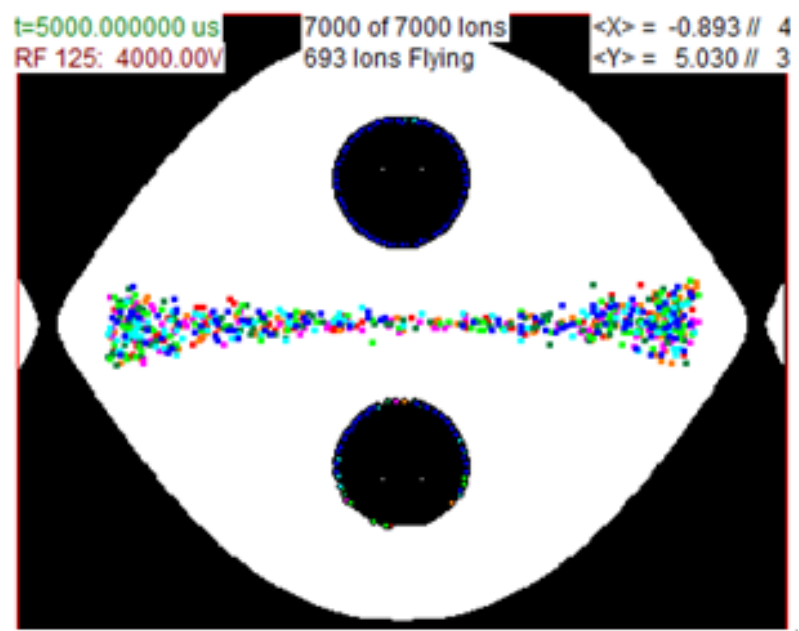

Fig. 6 a. Initial ion positions in the mid cross-section $(z=0)$ of a four-electrode harmonized Kingdon trap with merged electrodes as shown in Fig. 5, and b. their positions after $5 \mathrm{~ms}$ of flight (right). Ions have masses in the range from $50 \mathrm{Da}$ to 2,000 Da.

It is straightforward to show that the trap in question can work in the mode of both the Orbitrap and the Cassinian trap. However, most importantly, the trap with merged internal electrodes can hold ions near its center $(z=0)$ that were not necessary introduced from the outside; ions can be created directly inside the trap and accumulated there on oscillatory trajectories (Fig. 6). This feature is significant, it obviates the need to create a complex system of deflectors to input the ions from the outside; instead, ions can be created directly inside the trap by electron impact or photoionization. After creation, the ions begin to make stable oscillations between the internal electrodes in the direction of the coordinate perpendicular to the $z$-axis.

In order to measure the signal from the ions in a trap of this type, it is necessary to excite ion oscillations along its $z$-axis. The ions are not excited automatically, as is the case in the Orbitrap and in the Cassinian traps, in which ions are introduced from the outside across the $z$-axis with $z \neq 0$, where the potential value is not minimal and the ions begin to bounce along $z$ immediately after their introduction. It is possible to introduce ions from the outside of the merged two-electrode trap with a shift of the input point some distance from the center along the $z$-axis. However, in the mode of operation considered by us the ions are formed in the center of a trap with zero potential energy; therefore, a radiofrequency field resonant with respect to the harmonic oscillations along $z$ should be applied. Mass spectra measurement using the four-electrode trap with two merged electrodes can work as follows:

(i) first, ions are created by electron impact or photo ionization in the gap between the electrodes $(z=$ 0 ) and begin to make stable oscillations in the effective potential well in the $z=0$ plane;

(ii) then, when a sufficient number of ions are accumulated, their motion along the $z$-axis is excited by a broadband frequency-sweep signal [11] covering the whole range of frequencies of axial oscillations of trapped ions. To generate the dipole field frequency sweep, each of the internal electrodes is divided into two parts. 
(iii) The outer electrode is used to measure the induced currents, followed by its Fourier transform to obtain the mass spectrum. The external electrode is also divided into two parts insulated by radio frequency voltages, having the same electrostatic potential ( $0 \mathrm{~V}$ in this example).

A simulation of the first stage of operation of the merged-electrode trap as a mass spectrometer (ion accumulation) was conducted. The ions used in this simulation had a mass-to-charge ratio between 50 and 2,000 Da with homogeneous random distribution of initial positions in the range $0 \mathrm{~mm}<x<25$ $\mathrm{mm}$ and $0 \mathrm{~mm}<y<2 \mathrm{~mm}$; in addition, the ions had a thermal distribution of initial energies with an average value of $0.025 \mathrm{eV}$. The simulations predict that around $13.5 \%$ of the ions got trapped and have stable oscillations in $x-y$ plane.

A simulation of the second stage of operation of the merged-electrode trap as a mass spectrometer (excitation of axial oscillations of the ions) is shown on Fig. 7. The excitation is achieved with the help of a broadband frequency-sweep signal that is applied to the halves of the internal rods, for which they are cut in half as said previously. The frequency-sweep signal is a poly-harmonic signal containing the frequencies of trapped ions. In this simulation, the frequency-sweep signal was created by a special application, which first calculates the signal simply as a harmonic signal with a varying frequency and amplitude; the application then performs Fourier transform of the signal and removes all components of the spectrum that do not correspond to any of the captured ions. In the simulation, only the frequency range from $60 \mathrm{kHz}$ to $600 \mathrm{kHz}$ remains. Next, the application performs an inverse transformation of the received signal and saves its temporary implementation as a text file. The resultant frequency-sweep signal does not contain any unnecessary harmonics. For this simulation, the resulting frequency sweep signal had a duration of $13 \mathrm{~ms}$ and an actual maximum amplitude of $20 \mathrm{~V}$ (Fig. 7, top). As can be seen from the figure, with an increase in the frequency of the signal its amplitude decreases; this is necessary for a more uniform excitation of ions of different masses to the same amplitude of oscillations along the $z$ axis. The clustering of the ions based on their mass-to-charge ratio is shown in Fig. 7, bottom.

a.

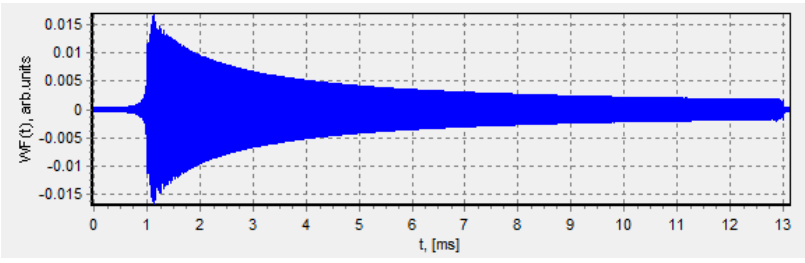

$$
\begin{aligned}
& t=20000.000000 \text { us } \\
& \text { RF } 125: 4000.00 \mathrm{~V}
\end{aligned}
$$

$b$.

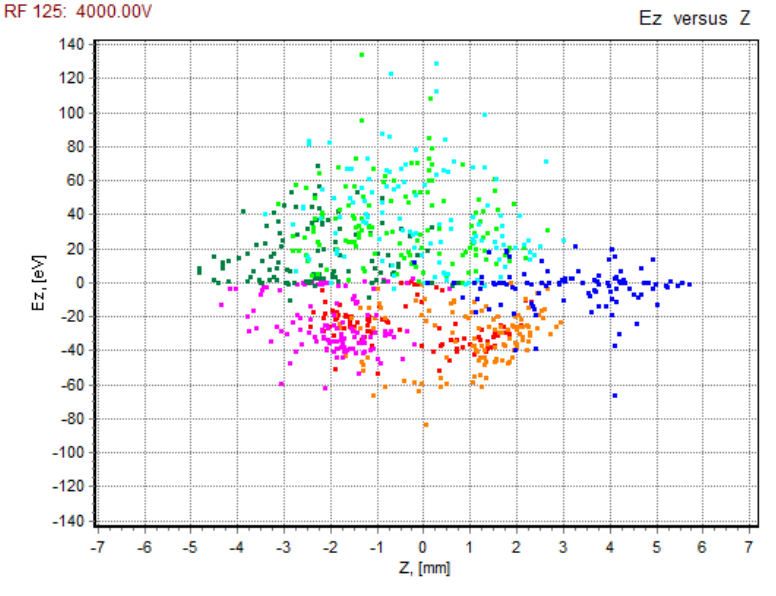


Fig.7. Amplitude versus time of the frequency-sweep signal (a.) and position of the ions in the $z$ - $E_{z}$ phase plane at the end of the frequency sweep excitation (b.). As initial conditions for ions, the coordinates and velocities obtained from the previous simulation of the ion accumulation process were used (Fig. 6). It can be seen that the ions oscillate with an amplitude of 5-6 mm, forming fairly compact groups based on their mass-to-charge ratio, which is a prerequisite for the successful registration of induced currents.

At the end of the frequency-sweep signal, the ions begin to perform harmonic oscillations along the $z$-axis, inducing a signal in the other pair of the external electrodes of the trap (third stage of operation of the merged-electrode trap as a mass spectrometer). Figure 8 shows the dependence of this signal on time during the first second. It can be seen that the signal of the induced current decreases somewhat with time; this is probably due to errors in integrating the equations of ion motion -neither collisions with gas nor field distortions in this simulation were taken into account in the simulations.

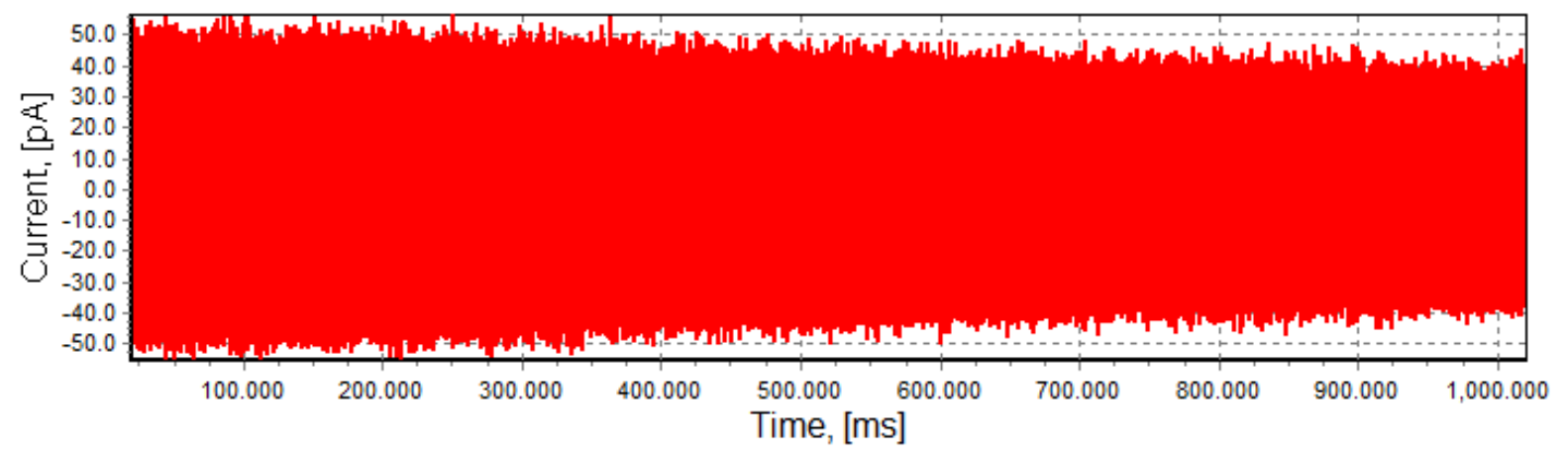

FFT of image signal. 2000000 samples. Resolution $\quad 0.95[\mathrm{~Hz}]$

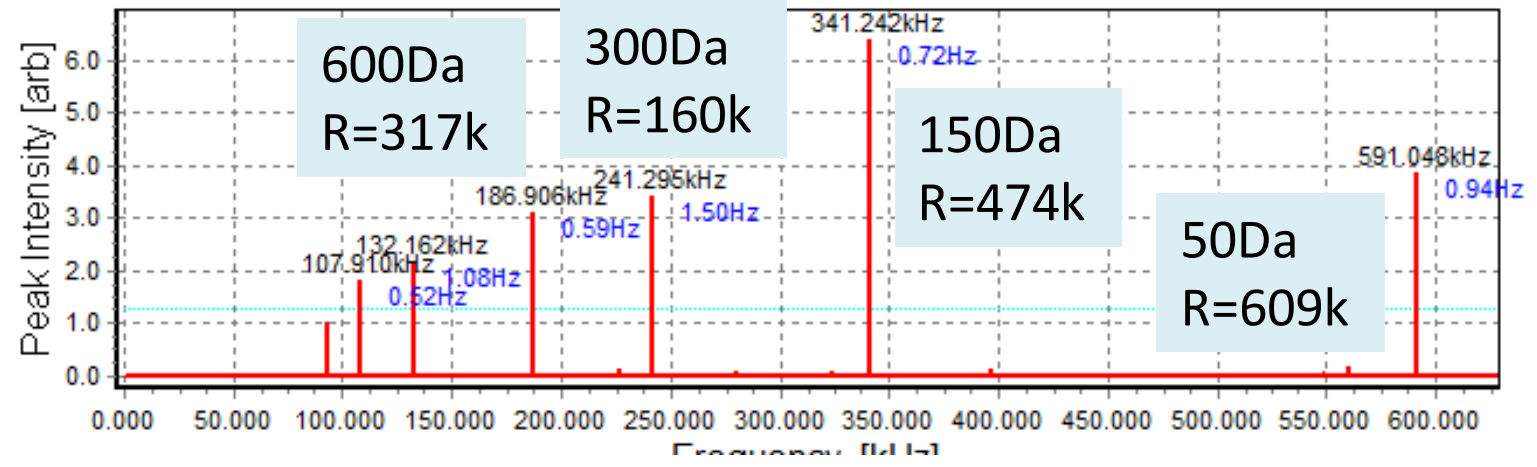

Fig. 8. Top: Current induced on the halves of the external electrode as a function of time. Bottom: Mass spectrum of trapped ions in the range from $50 \mathrm{Da}$ to 2,000 Da after conducting a Fourier transform (magnitude mode). For some of the peaks, the resolving power is indicated.

Figure 9 shows the mass spectrum of the trapped ions obtained by the Fourier transformation of the induced current as a function of time. For a mass-to-charge ratio of $50 \mathrm{Da}$, the resolving power is 609,000 ; this is a very high value -note that it was obtained without any apodization and other commonly used methods of Fourier spectrum processing. Using the existing techniques for processing spectra, the resolution can be doubled. As already mentioned, in this simulation the exact analytical field of the trap, calculated by a special application and stored in the PA format of the SIMION program files, was used. That is, the effects of truncation of the trap along the $\mathrm{Z}$ axis and the unevenness and inaccuracy of the electrode assembly were not taken into account. Of course, taking into account these factors, the result 
will not be so high, but we believe that with the development of technology these limiting parameters can be achieved.

\section{Conclusions}

Based on the analysis of the results of the study of various designs of multi-electrode harmonized Kingdon traps, we propose a new type of trap with two merged internal electrodes that has the ability to capture and accumulate ions formed inside. We show that a mass spectrometer with a relatively high resolving power can be created on the basis of such a trap. The creation of such traps requires the manufacture of complex electrodes, which is very expensive and time consuming if standard precision machining is used. However, with the advent of 3D printers [12] this becomes possible at a low cost and short production time. Additive manufacturing of our proposed trap with merged electrodes will require high-resolution manufacturing processes with associates materials (metals) that have high electrical conductivity, high-temperature compatibility (for system baking), and ultra-high-vacuum compatibility among other characteristics. Although the current accuracy and resolution of making metal electrodes via 3D printing is still not high enough [13],[14] to reach the limit values of the resolution of such devices as mass spectrometers, the technologies are constantly evolving and, undoubtedly, in the near future traps with curved electrodes, the principle of creation of which was first proposed by Y. K. Golikov, will be fully realizable and affordable.

\section{Bibliography}

[1] K. H. Kingdon, A method for the neutralization of electron space charge by positive ionization at very low gas pessures, Physics Review, vol. 21, pp. 408-418 (1923)

[2] S. Eliuk and A. Makarov, Evolution of Orbitrap mass spectrometry instrumentation, Annual Review of Analytical Chemistry, vol. 8, pp. 61-80 (2015)

[3] Y. K. Golikov, N. K. Krasnova, K. V. Soloviev, D. V. Nikitina, Integrable electrostatic ion traps, Applied physics (Russian) vol. 5, pp. 50-57 (2006)

[4] R. D. Knight, Storage of ions from laser produced plasmas, Appl. Phys. Lett. vol. 38, pp. 221-223 (1981)

[5] L. Gall, E.E. Pechalina, Y. K. Golikov, About one class of electrostatic fields with spatialtemporal focusing, Scientific instrumentation (Russian), vol. 24, pp. 18-26 (2014)

[6] D.V. Nikitina, Ion trap mass spectrometry in a dynamic mass spectrometry, thesis for $\mathrm{PhD}$ degree, St. Petersburg, 2006.

[7] C. Köster, The concept of electrostatic non-orbital harmonic ion trapping, Int. J. Mass Spectrom., 287, pp. 114-118 (2009)

[8] www.mssoft.pro

[9] http://simion.com

[10] https://www.ptc.com/en/products/mathcad/

[11] A. Marshall, T. L. Ricca, and T-C. L. Wang, Tailored excitation for trapped ion mass spectrometry, US patent 4,761,545 (1988)

[12] M. Vaezi, H. Seitz, and S. Yang, A review on 3D micro-additive manufacturing technologies, Int. J. Adv. Manuf. Technol., vol. 67, pp. 1721-1754 (2013) 
[13] Y. Zhong, L.E. Rannar, L. Liu, A. Koptyug, S. Wikman, J. Olsen, D. Cui, Z. Shen Additive manufacturing of 316L stainless steel by electron beam melting for nuclear fusion applications, J. Nucl. Mater., vol. 486, pp. 234-245 (2017)

[14] I. Gibson, D. Rosen, and B. Stucker, Directed energy deposition processes, Additive manufacturing technologies : 3D printing, rapid prototyping, and direct digital manufacturing, pp. 245-268 (2015).

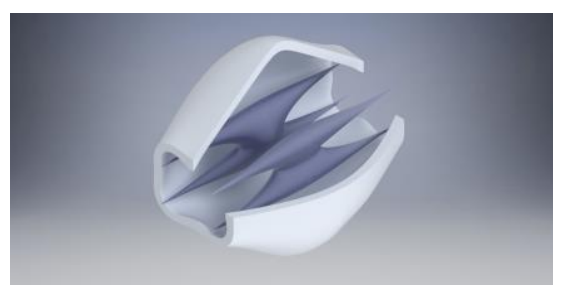

